\title{
Instrumentación de un irrigador para prueba VOR (reflejo vestíbulo ocular)
}

\section{Instrumentation of an Irrigator for Test VOR (Vestibule-Ocular Reflex)}

\author{
Campos-Cantón I. \\ Facultad de Ciencias \\ Universidad Autónoma de San Luis Potosí \\ Correo:icampos@fciencias.uaslp.mx \\ Castellanos-Velasco E. \\ Facultad de Ciencias \\ Universidad Autónoma de San Luis Potosi \\ Correo:ecastellv@fciencias.uaslp.mx
}

\author{
Martínez-Martínez J. \\ Facultad de Ciencias \\ Universidad Autónoma de San Luis Potosí \\ Correo: j_mtz_mtz@t-online.de
}

Información del artículo: recibido: julio de 2010, reevaluado: enero de 2011, aceptado: octubre de 2011

\section{Resumen}

En este artículo se realiza la instrumentación y automatización de un irrigador para prueba VOR (vestíbule ocular reflex). Esto se realiza mediante la irrigación de agua a tres temperaturas diferentes, en una o ambas fosas auditivas, en donde se estimula internamente cualquiera de los 3 canales semicirculares del sistema vestibular. Esto genera una respuesta Nistágmica medible a simple vista. El irrigador se forma mediante una instrumentación analógica acoplada a un PLC para su operación en automático.

\section{Abstract}

This paper shows one kind of VOR's (vestibule ocular reflex) implementation. Here, the VOR is built and designed with the instrumentation and automation of an irrigator system. This is done by water irrigation at three different temperatures, in one or both ear cavities, which it can stimulate internally any of the 3 semicircular canals of the vestibular system. This fact produces a measurable Nystagmic response at a glance. The irrigator is formed by analog instrumentation item coupled to a $P L C$, for its automated operation.

\section{Descriptores:}

- electrónica analógica

- prueba calórica VOR

- automatización

- amplificadores operacionales

- respuesta Nistágmica

\section{Keywords:}

- analog electronics

- test VOR

- automation

- operational amplifiers

- Nystagmic response 


\section{Introducción}

Este dispositivo surge de la necesidad de automatizar un proceso conocido como prueba de estimulación calórica descrita por Fitzgerald y Hallpike en 1942 (Kandel et al., 2000). El objetivo es la estimulación del sistema vestibular por medio de diferentes temperaturas, por ejemplo, a través de la inducción de agua (o viento en el caso de tener el tímpano roto), actuando ésta por convección en uno de los 3 canales semicirculares (normalmente es el canal semicircular horizontal) localizado en la parte baja del sistema auditivo, donde esta parte se denomina aparato vestibular (figura 1 ).

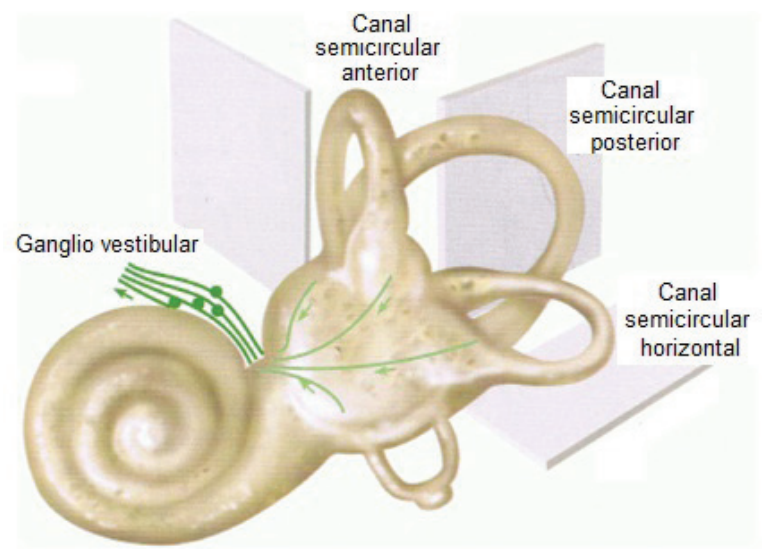

Figura 1. Canales semicirculares en el aparato vestibular

Existen trabajos previos como el realizado por Proctor, Metz y Dix (1976), en el cual se plantea la construcción de un aparato de estimulación por medio de aire. En nuestro caso, se elige el proceso de irrigación por medio de agua, ya que a este líquido se le pueden adicionar sustancias preventivas y/o terapéuticas como antisépticos o antibióticos, recetados por el otorrinolaringólogo.

Este procedimiento se utiliza para localizar lesiones en el tallo cerebral, pues aunque consume mayor tiempo y su aplicación es más difícil, es la que aporta más información.

Como el sistema vestibular guarda un gran sensor de posición en su interior (los canales semicirculares junto con el sáculo y el utrículo), su importancia radica en la correcta sensación de posición (tridimensional), velocidad angular y velocidad lineal que un individuo puede experimentar de su medio ambiente.

Esto se realiza básicamente con los siguientes pasos: se acuesta al paciente boca arriba y se procede a irrigar agua a una de las tres temperaturas establecidas $\left(20^{\circ} \mathrm{C}\right.$, $30^{\circ} \mathrm{C}$ y $44^{\circ} \mathrm{C}$ ), ya sea irrigando un oído o los dos (figura 2). Los volúmenes de agua establecidos son como mínimo de $200 \mathrm{ml}$ por un tiempo de $30 \mathrm{~s}$, dejando un lapso de 5 minutos entre cada prueba. Después de haber irrigado el o los oídos, se observa un reflejo medible llamado Nistagmo, el cual se traduce en un movimiento involuntario de los ojos. Este patrón de movimientos nistágmicos es el que da la información al médico sobre posibles lesiones o trastornos internos del paciente a nivel del tallo cerebral (pacientes con coma o vértigo).

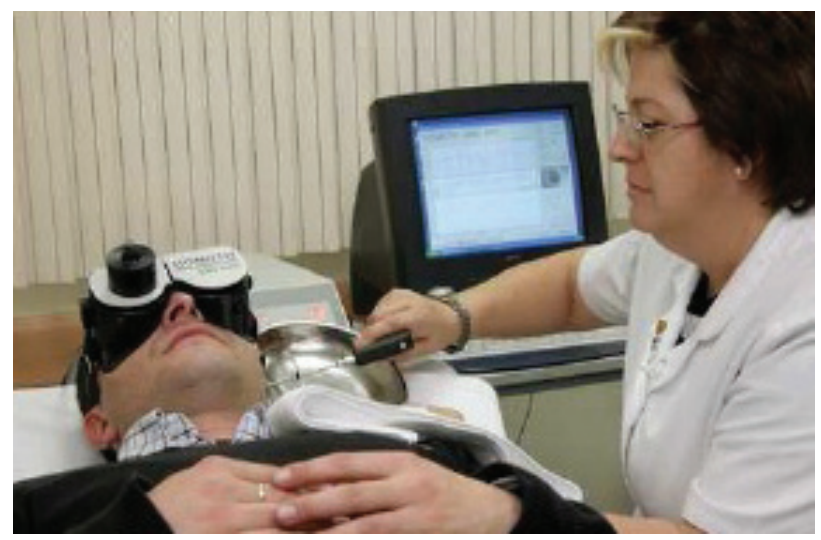

Figura 2. Procedimiento de irrigado con agua a una de las tres temperaturas establecidas $\left(20^{\circ} \mathrm{C}, 30^{\circ} \mathrm{C}\right.$ y $\left.44^{\circ} \mathrm{C}\right)$

A continuación se describe brevemente cómo está organizado el presente trabajo. En la sección de Métodos se muestran las expresiones matemáticas del circuito comparador. El arreglo experimental se observa en la sección correspondiente y finalmente se resumen las conclusiones.

\section{Métodos}

Para cumplir con los objetivos del probador VOR es necesario utilizar un comparador electrónico de instrumentación, en el que por una de sus terminales se coloca el potencial de referencia proporcional a la temperatura deseada y por la otra terminal de entrada se establece un potencial asociado a la temperatura real del agua. Una vez realizada la comparación se produce una señal en la terminal de salida, la cual sirve de referencia para activar un PLC. El PLC ejecuta un programa interno para activar o desactivar la corriente a través de una resistencia con la finalidad de calentar el agua a la temperatura deseada, y también por medio de su programación se decidirá cuándo iniciar el proceso de irrigación empleando una bomba de agua para ello.

El circuito comparador utiliza el amplificador operacional (amp op) de instrumentación ISL28470 de la compañía Intersil (2007), el cual es un amp op cuádruple diseñado para funcionar si es alimentado por una sola 
fuente en un rango de $2.4 \mathrm{~V}-5.5 \mathrm{~V}$ y con una variación de voltaje de entrada y salida dentro del rango de la fuente de alimentación (Rail-to-Rail input and output, RRIO). Este amplificador operacional de instrumentación es especial para mediciones en aparatos médicos e instrumentación industrial, su constitución interna se compone de arquitectura bipolar, como se aprecia en la imagen de la figura 3.

Utilizando las leyes de Kirchhoff se encuentra que el voltaje de salida obedece la siguiente relación en lazo abierto (Millman, 1979):

$V_{\text {out }}=2 A\left[\left(V_{1}-V_{2}\right)+\left(V_{4}-V_{3}\right)\right]$

Si se define $V_{I N}=\left(V_{2}-V_{1}\right), V_{3}=F B_{+}, V_{4}=F B_{-} \mathrm{y}$, ya que $A$ toma valores muy grandes, se tiene:

$V_{I N}=F B_{-}-F B_{+}$

De donde su configuración en lazo cerrado se muestra en la figura 4.

Por lo tanto, las ecuaciones que rigen el comportamiento del circuito de la figura 4 son:

$$
\begin{aligned}
& F B_{+}=V_{R E F} \\
& F B_{-}=V_{\text {out }} \frac{R g}{\left(R_{g}+R_{f}\right)}, \\
& V_{I N}=V_{\text {out }} \frac{R g}{\left(R_{g}+R_{f}\right)}-V_{R E F} \\
& V_{\text {out }}=\left(V_{\text {IN }}-V_{\text {REF }}\right)\left(1+\frac{R_{f}}{R_{g}}\right)
\end{aligned}
$$

El voltaje de referencia $V_{\text {REF }}$ se toma del sensor de temperatura LM35 de la marca National Semiconductor, el cual está configurado para proporcionar $10 \mathrm{mv} /{ }^{\circ} \mathrm{C}$; si utilizamos $R_{f}=200 \mathrm{k} \Omega$ y $R_{g}=470 \Omega$ en (3) se garantiza un error de $\pm 1^{\circ} \mathrm{C}$ en el control de la temperatura. En este caso se requiere que el dispositivo sea capaz de calentar el agua a $20^{\circ} \mathrm{C}, 30^{\circ} \mathrm{C}$ y $44^{\circ} \mathrm{C}$, es decir, los potenciales sensados por el LM35 serán de $200 \mathrm{mV}, 300 \mathrm{mV}$ y $440 \mathrm{mV}$, respectivamente. Por ejemplo, si el irrigador tiene una temperatura de $17^{\circ} \mathrm{C}$ y se desea una temperatura de $20^{\circ} \mathrm{C}$, se debe encender la resistencia calefactora hasta que el agua alcance los $20^{\circ} \mathrm{C}$. Así, utilizando la ecuación 3 se tiene: $V_{I N}=170 \mathrm{mV}$ y, por conveniencia para la instrumentación electrónica se utiliza lógica negativa para la señal de referencia $V_{R E F}=-200 \mathrm{mV}$.
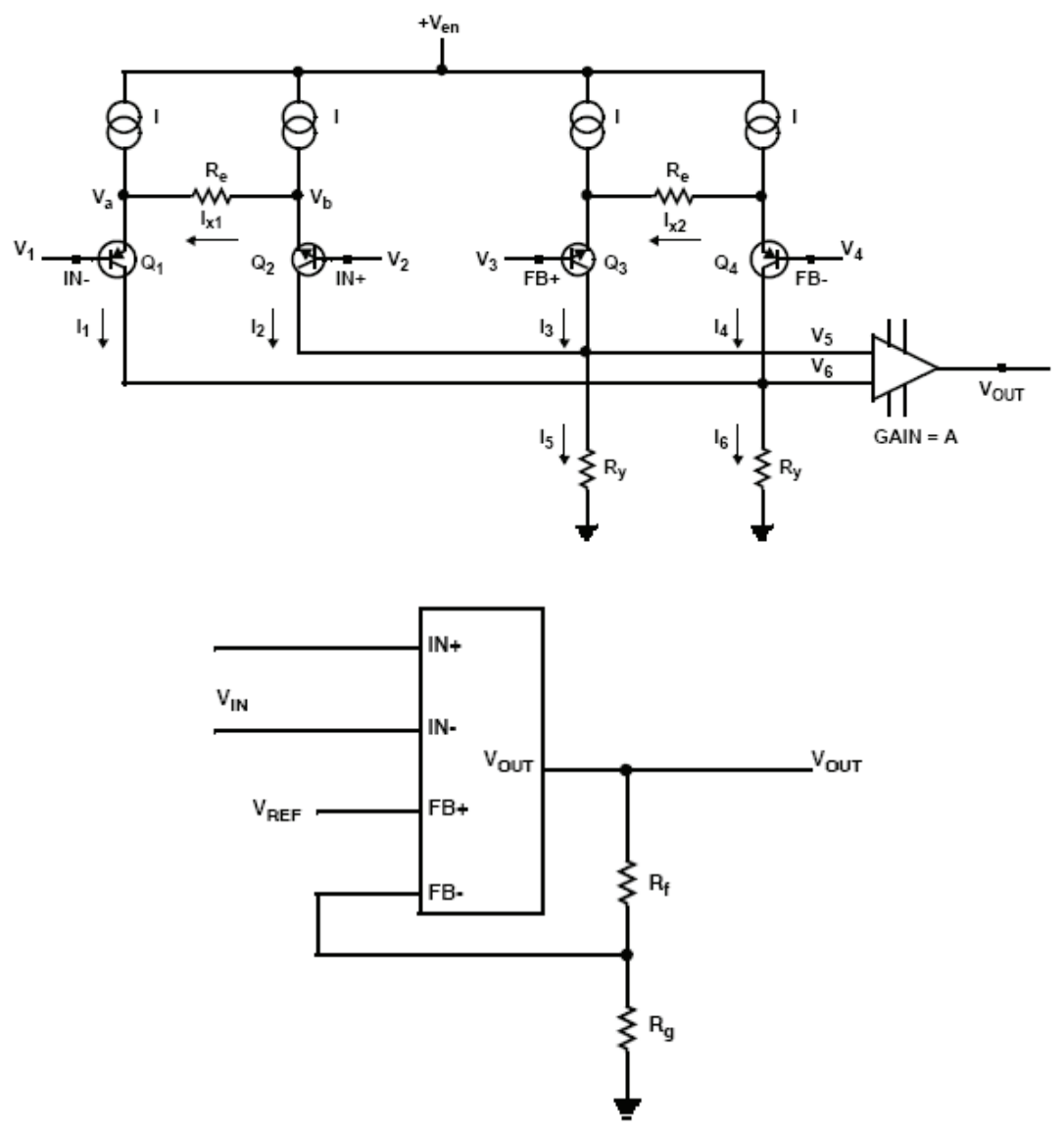

Figura 3. Diagrama esquemático interno del circuito de instrumentación ISL28470 para comparar las diferentes temperaturas con la deseada 


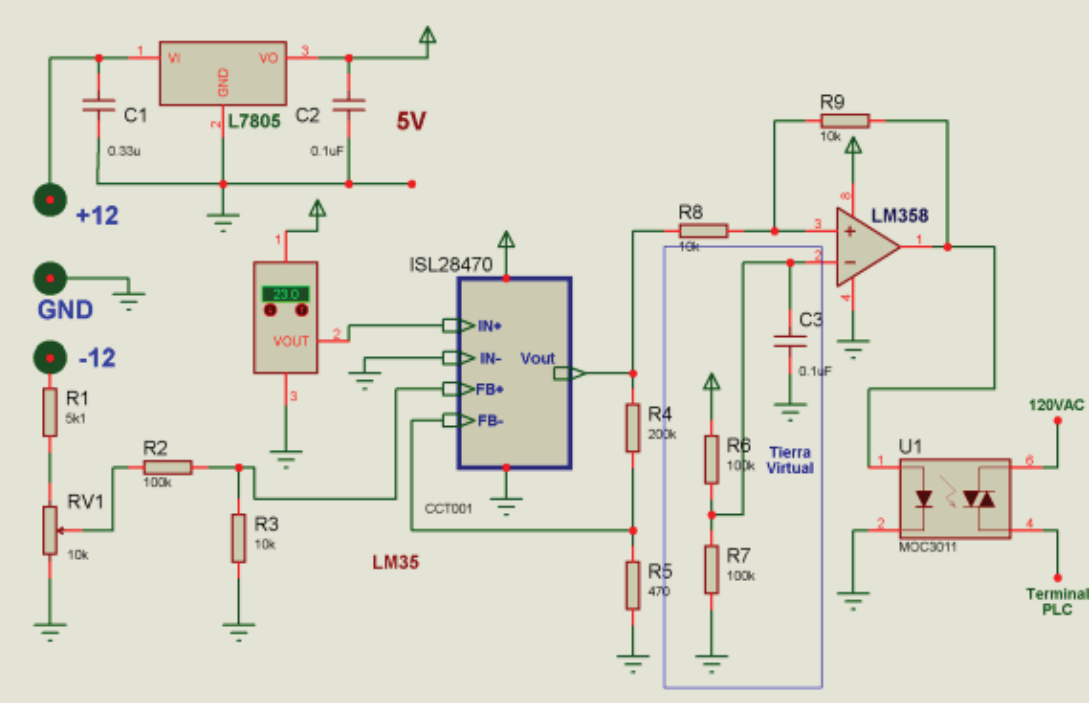

Figura 5. Circuito de acoplamiento para el PLC que incluye fuente de voltaje, sensor de temperatura y circuito comparador

$$
V_{\text {out }}=(170 m-200 m)\left(1+\frac{200 k}{470}\right)
$$$$
V_{\text {out }}=-12.79 \mathrm{~V}
$$

Entonces $V_{\text {out }}=0 \mathrm{~V}$ por RRIO. Con este potencial de salida la resistencia de calefacción se encuentra en estado apagado, por lo que es necesario el efecto contrario, esto se logra invirtiendo la señal, lo cual se realiza con el LM358 y el MOC3011(figura 5).

\section{Arreglo experimental}

Como se mostró en la sección anterior, la figura 5 sirve de transductor con entrada a la temperatura real del agua y con salida a una señal de corriente alterna. Esto por la necesidad de interacción con el PLC (Manual Logo, 2003) (figura 6). El diseño se basa en realizar tres circuitos como el mostrado en la figura 5, cada uno compara de manera independiente la temperatura de $20^{\circ} \mathrm{C}, 30^{\circ} \mathrm{C}$ y $44^{\circ} \mathrm{C}$, los circuitos se conectan en las terminales I2, I3 y I4 del PLC, respectivamente; asimismo, se tienen conectadas las entradas I5 a un interruptor SW1, I6 a SW2, I7 a SW3 e I8 a SW4, que son las señales para activar las temperaturas de $20^{\circ} \mathrm{C}, 30^{\circ} \mathrm{C}, 44^{\circ} \mathrm{C}$ y el botón de inicio de irrigación, respectivamente. El PLC contiene dos programas internos específicos para controlar dos salidas conectadas a una resistencia calefactora Q1 (la cual calienta el agua) y una bomba de succión Q2 (que succiona y entrega agua a presión regulable por 30 segundos), vea la parte inferior de la figura 6. Estos programas se muestran en las figuras $7 \mathrm{a}$ y $7 \mathrm{~b}$, que son el control de la temperatura y el inicio de irrigación. El aparto cuenta con una sonda de polietileno desechable para uso individual (se puede implementar una sonda semejante al otoscopio si así se requiere) y cuenta con manguerillas de HMW-HDPE (polientileno de alto impacto).

El diseño implementado por el PLC permite manipular directamente valores lógicos de señales con voltajes alternos entrada/salida (110/220 Vca), necesarios para accionar la lógica del PLC y los actuadores. A la vez, este proceso de automatización se vuelve flexible, ya que es posible reprogramar el PLC sin necesidad de rediseñar completamente el sistema (figura 8). El protocolo de calibración se realizó de acuerdo al protocolo propuesto por la Sociedad Británica de Audiología (2010).

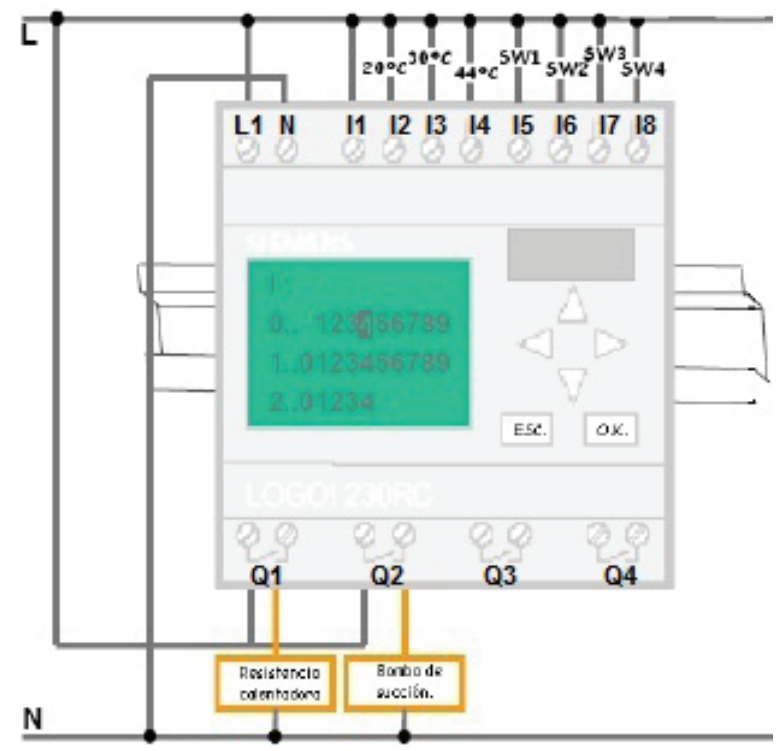

Figura 6. Conexiones externas al PLC, donde $\mathrm{I}_{2,3,4}$ entradas de temperatura; $I_{5,6,7,8}$ interruptores de inicio de operación y $Q_{1,2}$ 
a)

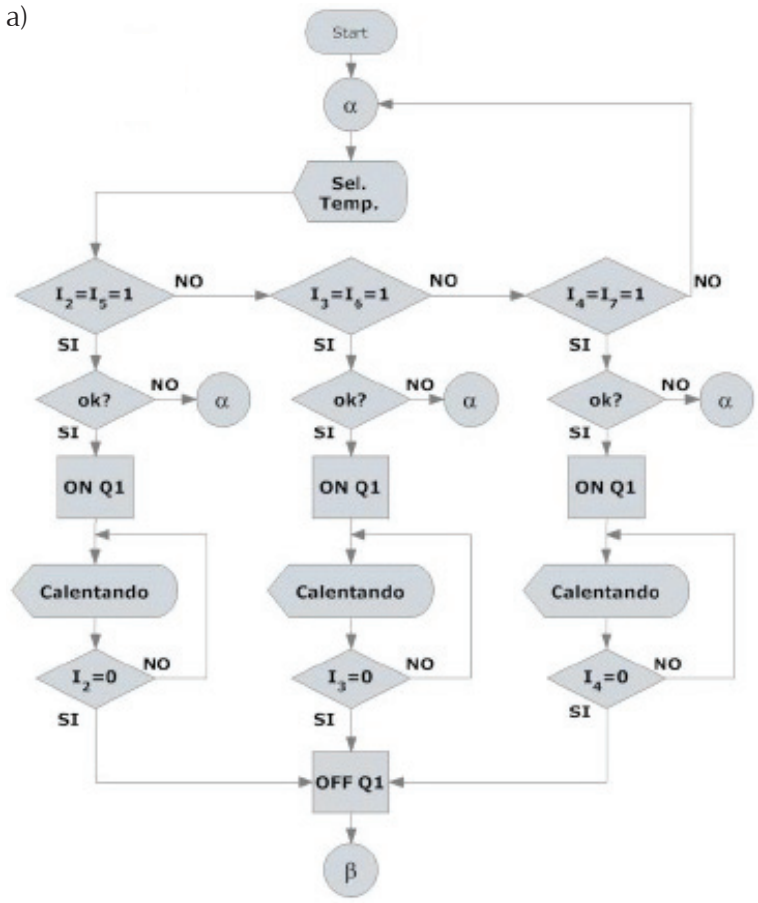

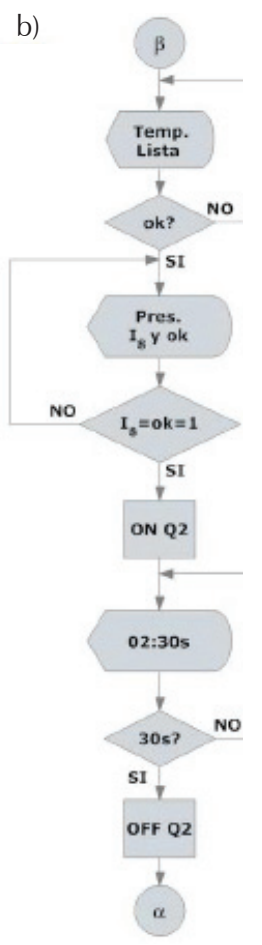

b)

Figura 7. Diagrama de flujo del programa implementado por el PLC; a) control de temperatura y b) control de irrigación

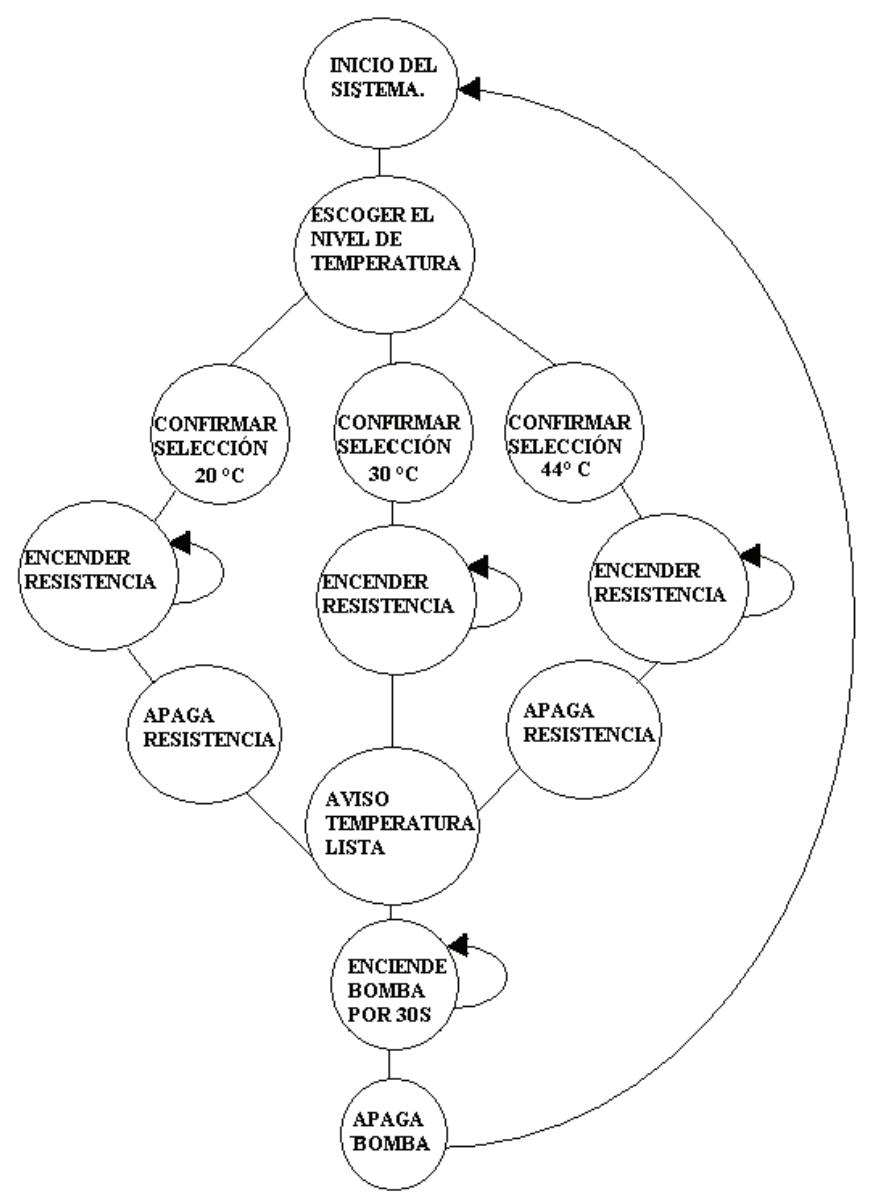

Figura 8. Grafo del autómata que describe el funcionamiento del sistema 


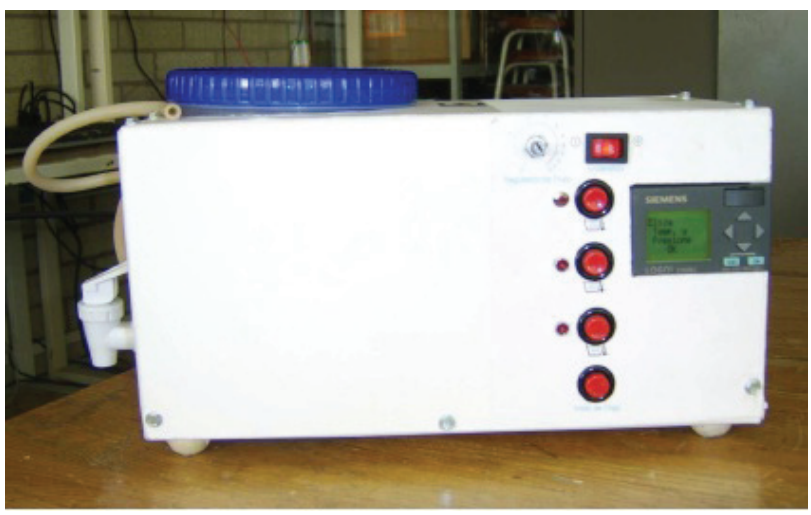

Figura 9. Vista externa del prototipo del irrigador VOR, en la parte superior está la tapa del tanque de agua, botones de selección de temperatura en color rojo y en la parte derecha se encuentra el PLC

\section{Conclusiones}

Se realizó la instrumentación de un irrigador para prueba VOR (vestíbulo ocular reflex), con el uso de un PLC LOGO!230RC como etapa de potencia y decisión, asimismo un circuito electrónico auxiliar para el proceso de sensado de la temperatura. El irrigador (figura 9) permite la automatización de un proceso necesario en el diagnóstico de desórdenes en el equilibrio o, en el peor de los casos, para diagnosticar lesión en el tallo cerebral de pacientes en coma.

El procedimiento sin la automatización no estipula una temperatura específica (examen rutinario) sólo estima respuesta para temperatura 'fría' o 'caliente', resultando en un procedimiento lento, debido a que el calentamiento del agua se lleva a cabo manualmente en una estufa.
Este irrigador es un prototipo experimental para su continua mejora.

\section{Referencias}

Kandel R.E. et al. Principles of NeuralScience, 5a ed., Nueva York, USA, McGraw-Hill, 2000.

Proctor-Leonard M.D. et al. Construction of a Practical and Inexpensive Air Stimulator for Caloric Vestibular Testing. The Laryngoscope [en línea] volumen 86 (número 1), enero de 1976: 126-131. Disponible en: http://onlinelibrary.wiley.com/ doi/10.1288/00005537-197601000-00024/abstract

ISL28270, ISL28273, ISL28470, Application Note AN1298,2. Calif.,USA. Intersil Americas Inc., 2007. AN1298.2

Millman J. Microelectronics Digital and Analog Circuits and Systems, 3a ed., McGraw-Hill, 1979.

Manual LOGO! Neurnberg Deu. Bereich Automation and Drives. Siemens AG, 2003.

Recommended Procedure for Caloric Test. United Kingdom, British Society of Audiology, 2010.

\section{Este artículo se cita:}

\section{Citación Chicago}

Campos-Cantón, Isaac, Ernesto Castellanos-Velasco, Jorge Martínez-Martínez. Instrumentación de un irrigador para prueba VOR (reflejo vestíbulo-ocular). Ingeniería Investigación y Tecnología XIII, 04 (2012): 433-438.

\section{Citación ISO 690}

Campos-Cantón I., Castellanos-Velasco E., Martínez-Martínez J. Instrumentación de un irrigador para prueba VOR (reflejo vestíbulo-ocular). Ingeniería Investigación y Tecnología, volumen XIII (número 4), octubre-diciembre 2012: 433-438.

\section{Semblanza de los autores}

Isaac Campos-Cantón. Recibió el grado de maestro en ingeniería en 1997 por la Facultad de Ingeniería de la UNAM y el grado de doctor en el 2009 por el IICO-UASLP. Actualmente es profesor de tiempo completo en el área de electrónica de la Facultad de Ciencias, UASLP. Sus áreas de interés son la instrumentación y desarrollo de circuitos electrónicos con comportamiento no lineal.

Ernesto Castellanos-Velasco. Recibió su licenciatura en electrónica y comunicaciones en 1999 por la Universidad Iberoamericana, el grado de maestro en ingeniería eléctrica en 2001 y el grado de doctor en control automático en 2004, ambos por el CINVESTAV-IPN, México. Actualmente es profesor en el Departamento de Electrónica en la Facultad de Ciencias, UASLP. Sus áreas de interés son el análisis y síntesis de sistemas en tiempo discreto, teorías de control y el estudio de instrumentación e interfases.

Jorge Martínez-Martínez. Se tituló con la licenciatura en electrónica por la Facultad de Ciencias, UASLP en enero del 2010, Su área de interés es la instrumentación de circuitos electrónicos. 